\title{
Quality of experience evaluation of voice communication: an affect-based approach
}

\author{
Abhishek Bhattacharya ${ }^{1 *}$, Wanmin $\mathrm{Wu}^{2}$ and Zhenyu Yang ${ }^{1}$
}

\author{
* Correspondence: abhat002@cis. \\ fiu.edu \\ ${ }^{1}$ School of Computing and \\ Information Sciences, Florida \\ International University, Miami, FL \\ 33199, USA \\ Full list of author information is \\ available at the end of the article
}

\begin{abstract}
Voice communication systems such as Voice-over IP (VoIP), Public Switched Telephone Networks, and Mobile Telephone Networks, are an integral means of human tele-interaction. These systems pose distinctive challenges due to their unique characteristics such as low volume, burstiness and stringent delay/loss requirements across heterogeneous underlying network technologies. Effective quality evaluation methodologies are important for system development and refinement, particularly by adopting user feedback based measurement. Presently, most of the evaluation models are system-centric (Quality of Service or QoS-based), which questioned us to explore a user-centric (Quality of Experience or QoE-based) approach as a step towards the human-centric paradigm of system design. We research an affect-based QoE evaluation framework which attempts to capture users' perception while they are engaged in voice communication. Our modular approach consists of feature extraction from multiple information sources including various affective cues and different classification procedures such as Support Vector Machines (SVM) and k-Nearest Neighbor (kNN). The experimental study is illustrated in depth with detailed analysis of results. The evidences collected provide the potential feasibility of our approach for QoE evaluation and suggest the consideration of human affective attributes in modeling user experience.
\end{abstract}

\section{Introduction}

The voice communication industry is undergoing a rapid phase change with technologies such as cellular, mobile and Internet telephony replacing the conventional telephone networks. Service providers are faced with offering high communication quality under more heterogeneous and dynamic networking conditions. Effective evaluation of system performance is becoming critical, which will serve as an important instrument for quality monitoring and management.

Traditional evaluation methods are very system-oriented where Quality of Service (QoS) metrics have been the de facto standards for voice communication technologies.

Recently, there is a paradigm shift towards user-oriented methodologies with the introduction of human-centric computing in the systems area [1], and the concept of Quality of Experience (QoE) is gradually gaining popularity [2-4]. Since QoE metrics are closely related to human perception, they could potentially serve as more valuable quality indicator from the user perspective.

The main challenge of how to evaluate QoE remains largely unsolved due to its complexity. Over the years, different meanings have been attached to the term [5-7].

(c) 2012 Bhattacharya et al; licensee Springer. This is an Open Access article distributed under the terms of the Creative Commons Attribution License (http://creativecommons.org/licenses/by/2.0), which permits unrestricted use, distribution, and reproduction in any medium, provided the original work is properly cited. 
Theoretical studies from various disciplines characterize $Q o E$ as a multi-dimensional construct which involves both subjective and objective factors intertwined in the user interaction such as perception, emotion, behavior, need, context, system and networking [8-14]. In practice, system developers have applied QoE assessment techniques, ranging from user feedback $[15,16]$, QoS-based estimation $[17,18]$ to media quality analysis $[19,20]$. Despite the value demonstrated, each approach has limitations and weaknesses as elucidated in related work (Related work).

In this paper, we investigate the usability of affective computing in evaluating QoE of voice communication. Affective computing deals with the analysis of human emotional variables naturally revealed during the user-system interaction. In the process, emotions have been shown to have strong association with user experience regarding interest, satisfaction, motivation and performance [21-24]. Using signal processing, linguistic analysis, and psycho-physiological techniques, automated emotion recognition is feasible by aggregating affective cues from multi-modal user input such as facial expression, speech, body gesture, and neuroimaging [25-27]. Leveraging on these findings, affect-aware systems are emerging that dynamically adapt according to the change of user emotions in applications of user interface, health care, education, customer service, intelligent automobile, entertainment, information retrieval and social signal processing [28-33].

Guided by the above evidences, we hypothesize that QoE or the user perception of quality in voice communication is correlated to his/her affective behavior (e.g., pitch, voice, timing and articulation), which will vary across networking conditions. To the best of our knowledge, the analysis of affective behavior and its role in the QoE evaluation of voice communication is an unexplored area. As an initial step, we focus on experimental user studies to record changes of user affect state and examine at what level QoE is reflected from these changes.

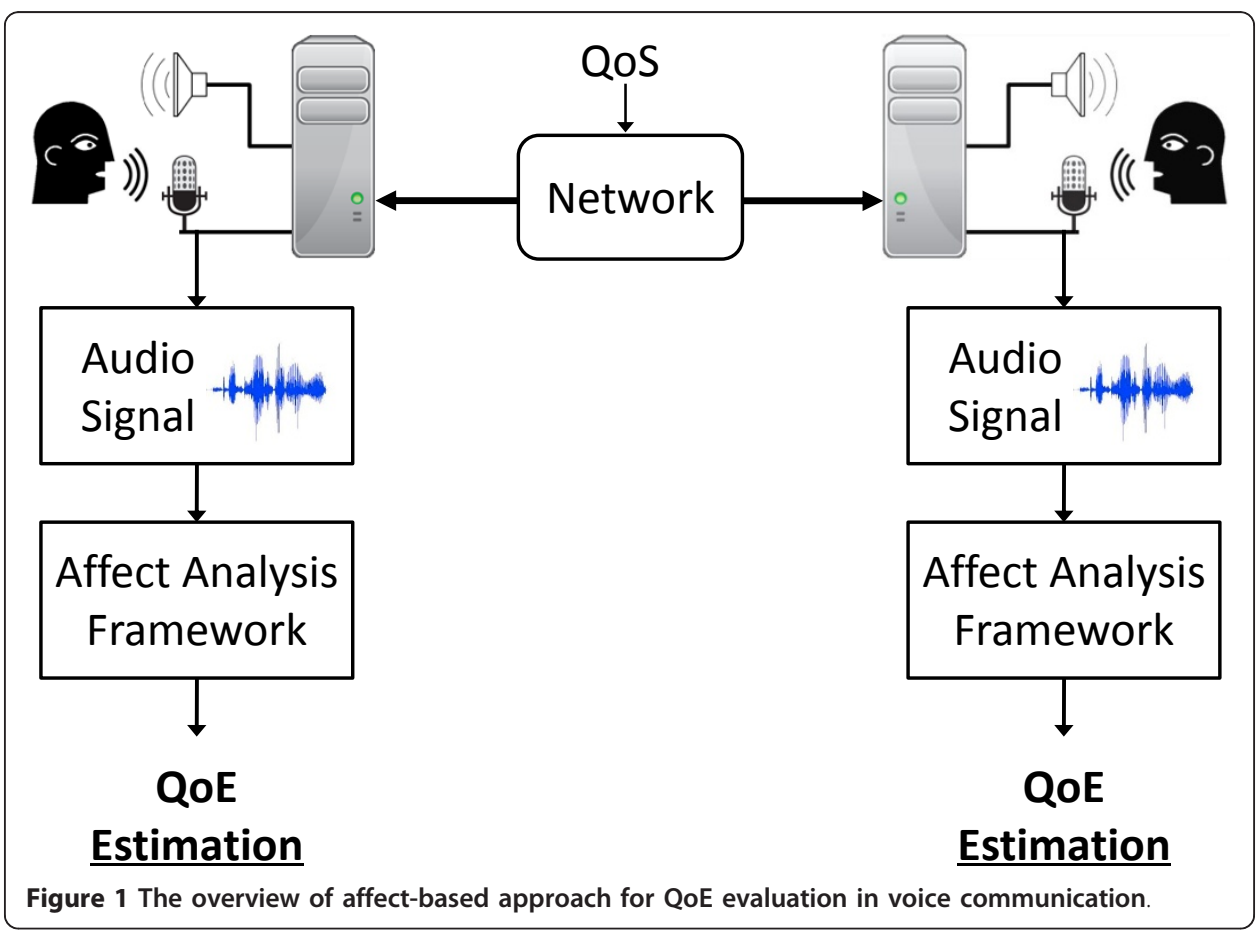


The general scheme of the experiment is given in Figure 1, which shows the network setting, the voice communication, the audio signal input, and the affect analysis framework that processes audio signals to produce QoE estimation.

Our study is based on the context of human-human voice communication. In a test session, two participants are engaged in natural conversation through a VoIP connection mediated with varying QoS parameters (e.g., delay, loss rate, and bandwidth). During the conversation, the communication quality is tagged by user feedback ("Good", "Average" and "Bad"). The conversation is saved and further processed by the affect analysis framework offline. The front end of the framework performs feature extraction to derive samples. The set of features are drawn from three major categories of affective signals involving acoustic, lexical and discourse features as suggested by previous research in emotion detection [25,34-36]. The affective behavior is analyzed individually for each category as well as combined. A subset of samples are used to train classification modules. Then, the rest samples are used for performance testing. Finally, the accuracy analysis is provided by comparing the output of the affect analysis framework (i.e., QoE estimation) with the user tagged feedback.

Our contributions in this paper are the followings. Most of all, we provide a new affect-based methodology of QoE evaluation in voice communication. Different from previous approaches, we propose to assess quality directly from the user affective responses. Therefore, our method has the advantage of deriving subjective QoE measures in an implicit and non-intrusive manner. The experimental results indicate very promising prospect of this approach. Regarding boarder impacts, as the communication systems become more media-rich and interactive (e.g., spatial audio, 3D/immersive space), measuring QoE via indirect methods will become more challenging [4]. Therefore, our work represents an important step towards the understanding of QoE for the future generation of communication systems.

In the remainder of the paper, Related work reviews existing work on QoE evaluation in voice communication. Experimental design describes the methodologies and settings of the user study experiment. Affect analysis framework presents the details of the affect analysis framework. Results highlights the results and implications of affectbased approach on the prediction of QoE. The paper is concluded in Conclusions with discussion on future directions.

\section{Related work}

We classify available QoE evaluation methods of voice communication in three groups: (a) user feedback, (b) QoS-based estimation, and (c) media quality analysis.

\section{User feedback}

These group of methods obtain explicit input from user for quality measurement. For example, in the popular format of Mean Opinion Score (MOS) [16], users are asked to complete a questionnaire based on a 1-to-5 scale. It is a simple method that provides subjective measures of user perception. The main disadvantage is its intrusiveness [29]. The laboratory settings are often not transparent to the participants, which destroys the eco-psychological validity of a naturalistic study. Thus, the user feedback may not easily elicit spontaneous expressive behavior. Another disadvantage is the issue of scaling quality with numbers $[37,38]$. To alleviate the problem, an interesting idea of One 
Click [15] is proposed where the user only needs to click the mouse whenever he/she feels dissatisfied with the quality. Compared with traditional user feedback, it is less intrusive: the user task is reduced from a multiple-choice decision to a dichotomous one, and the test can be performed "during" user interaction instead of "after." However, One Click still requires direct user attention which poses cognitive overhead. For more interactive systems like gaming or 3D immersion, it implies that the user may have to pause his/her ongoing activity from time to time.

\section{QoS-based estimation}

A good amount of research has been done in this area [17,18,39-41], where QoE is estimated by modeling from the underlying QoS parameters. QoS-based methods are implicit and non-intrusive which makes them an appealing choice. However, these methods are essentially objective approximation of QoE due to lack of user engagement. Accordingly, they cannot cover all QoE dimensions that may affect user perception and experience [15], and the discrepancy among the user population tend to be ignored [42]. For example, users have different sensitivity to delays under varying conversational dynamics (e.g., various talk/silent spurt duration) [18]. It is hard to accommodate such feature in QoS-based estimation. The correlation between QoS and QoE also becomes more intractable for advanced communication systems, which greatly complicates the modeling process [7]. Moreover, there are non-trivial technical details regarding its deployment in the field such as messaging overhead and traffic detection $[43,44]$, and the buffer masking effect on the interaction between QoE and delay/loss [40].

\section{Media quality analysis}

Media quality analysis methods assess the quality by measuring the distortion of the signal based on certain analysis models like signal-to-noise ratio (SNR). More sophisticated ones attempt to incorporate human auditory perception such as Enhanced Bark Spectral Distortion (EBSD) and Perceptual Speech Quality Measure (PSQM) [45]. Similar to QoS-based estimation, these methods do not require explicit user input. The state-of-the-art standard in this category is ITU-T P.862 [19], also known as the Perceptual Evaluation of Speech Quality (PESQ). The drawback of PESQ is that it is double-ended: the algorithm requires both the original and the degraded signals to compute the quality difference [46]. Further, it fails to consider factors such as various listening levels, sidetone/talk echo, and conversational delay/interaction [15,17].

We argue that a suitable QoE evaluation method in voice communication should capture the subjective measures from the user in a non-intrusive manner. It is clear that none of the existing methods comprises of both characters. The contribution of this paper is the proposition of a new affect-based approach which opens such possibility.

\section{Affect analysis framework}

The basic approach of affect-based framework is to apply multi-modal analysis for QoE evaluation. The front end of the framework performs feature extraction from the audio signals of user conversation. In our study, three major categories of affective signals are extracted including acoustic features, lexical features and discourse features. 
The importance and usage of acoustic features for automatic emotion detection has been well-studied [34]. However, acoustic-based methods are effective for posed expressions in staged scenarios but degrades in natural human interaction with spontaneous expression [25]. The common wisdom is to incorporate acoustic features along with lexical and discourse features for performance enhancement [25,35]. Since we target normal human-human voice communication, we include all these features in the framework. After feature extraction, the affective response is analyzed individually by the classification module of each category. In the last stage, the results are then combined to generate the final output of the framework (i.e., QoE estimation).

We follow the general methodologies and technical justifications as presented in [36] for the design of the framework, but we independently completed the implementation and integration work. We briefly introduce each module of the framework in the following sections as it is not our major focus of this research. An overview of the framework is presented in Figure 2.

\section{Acoustic features}

We consider 22 different acoustic attributes related to segmental and suprasegmental information of speech signals. These attributes are derived from turn-level statistics and transformations in the domains of fundamental frequency, energy, duration, and formants.

- Fundamental Frequency (FO): the lowest frequency of the signal wave. We use statistical functionals of mean, median, standard deviation, maximum, minimum, range (maximum-minimum) and linear regression coefficient.

- Energy $\left(E_{r}\right)$ : computed as the Root Mean Square (RMS) of the signal, i.e., $E_{r}=\sqrt{\frac{\left(\sum_{n=0}^{N} x_{n}^{2}\right)}{N}}$ a PCM frame of size $N$. Similarly, we use statistical functionals of mean, median, standard deviation, maximum, minimum, range, and linear regression coefficient.

- Duration: computed by comparing various voiced and non-voiced regions in a temporal-domain analysis of the speech waveform. The individual attributes are speechrate, duration of the longest voiced speech and ratio of voiced and unvoiced region.

- Formants: basically the resonance of human vocal tract. Formant location and bandwidth are used to identify phonetic property of human speech. We use the first and second formant frequencies $(F 1, F 2)$, their corresponding bandwidths $(B W 1, B W 2)$ and the mean.

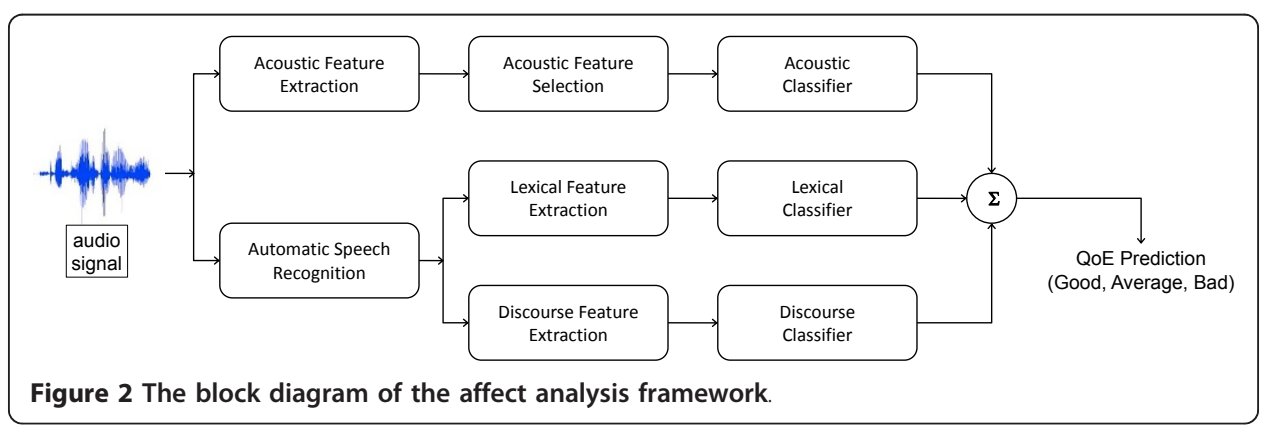


The module of acoustic feature extraction was implemented based on the tool of openSMILE [47], an open-source software that can extract many low-level acoustic features and statistic functionals. The 22 acoustic attributes form the base feature set (denoted as Base). Generally, not all features are equally significant for affective analysis. Following the work of [36], we perform feature selection with an expectation to improve prediction accuracy by dropping off the least significant attributes. We employ the leave-one-out method for feature selection and the nearest neighborhood rule for estimating accuracy. We generate two subsets from the base feature set, one has 10 best features (denoted as f10) and the other has 15 best features (denoted as f15). Lastly, we obtain another feature set by performing Principal Component Analysis (PCA) on the base feature set, which is denoted as PCA.

\section{Lexical features}

Lexical features here refer to language related information regarding the fact that people tend to choose specific words for various expressions (e.g., "can't", "damn", "bad", and "great") [9]. In our study, we adopt the notion of mutual information [48] to establish the correlation between words and different $Q$ o E levels.

Denote the vocabulary $\mathrm{V}=\left\{V_{1}, V_{2}, V_{n}\right\}$ and the set of quality levels $Q=\left\{q_{1}, q_{2}, q_{m}\right\}$. As mentioned earlier, we choose $\mathrm{m}=3$ (i.e., "Good", "Average", and "Bad"). The mutual information is given as:

$$
I(v, q)=\log \frac{\operatorname{Pr}\{q \mid v\}}{\operatorname{Pr}\{q\}}
$$

for $v \in V$ and $q \in Q$. Intuitively, if a word $\mathrm{v}$ is correlated with a quality level $q$, then $\operatorname{Pr}\{q \mid v\} \operatorname{Pr}\{q\}$ and $I(v, q)$ is positive. If there is no correlation, then $\operatorname{Pr}\{q \mid v\}=\operatorname{Pr}\{q\}$ and $I(v, q)$ is zero. If $\mathrm{v}$ makes $q$ less likely, $I(v, q)$ is negative.

Given an utterance, the action for quality level $q_{k}$ can be calculated as:

$$
a_{k}=\sum_{i=1}^{n} O_{i} I\left(q_{k}, v_{i}\right)+\log \left(q_{k}\right)
$$

where $O_{i}$ is the likelihood that word $V_{i}$ is recognized in the utterance by the speech recognition module. For simplicity, we use a one-layer network where the order and sequence of words are not considered. Finally, we use $\left\{a_{0}, a_{1}, a_{2}, a_{0},-a_{1}, a_{1}-a_{2}, a_{2}-a_{0}\right\}$ as the feature set for the lexical information. The intent of this module is to associate indicative words with respect to the different quality categories which will help for better prediction. From experimental voice data, we found that participants used words such as "Damn", "Disgusting" more frequently whenever they rated the quality as "Bad." This correlation is automatically captured by lexical module whereby these words will be marked as indicative for corresponding quality category to improve prediction accuracy.

We leverage on the Automatic Speech Recognition (ASR) system from the HTK Toolkit [49] of Cambridge University for predicting text output of voice signals, which is based on the Hidden Markov Models (HMM). The models of HMM are accessed and trained on Wall Street Journal (WSJ) corpora [50] and the generated tied-state cross-word triphones are utilized for later recognition purposes. We develop a 3-gram language model provided in a 1,200 million English Gigaword corpus citecorpus 
indexed with the Linguistic Data Consortium catalogue and also coupled with a 125,000-word CMU pronunciation dictionary [51].

\section{Discourse features}

The value of discourse information for emotion recognition has been noted in the literature, particularly combined with acoustic information to improve the accuracy $[52,53]$. In our study, we take a simplified approach by modeling only repetition, which is found to be the most important indicator of trouble in communication [35]. We choose from 1-word to 5-word repetitions and formulate the dimensions of discourse feature set as: number of 1-word repetition, number of 2-word repetitions, and likewise. Moreover, we construct another repetition metric as the following:

$$
R=\sum_{i=1}^{5} r_{i} * w_{i}
$$

where $r_{i}$ is the number of $i$-word repetitions and $w_{i}$ represents a proportional weightage which assigns higher weight to the repetition of longer sequence of words (e.g., 5-word repetition).

Analysing experimental voice data, we found that participants used words such as "not able to hear", "no sound", "pardon me", and "please repeat" more frequently when they rated the quality as "Bad." This correlation between the particular repetitive words and quality category label is automatically analyzed by the discourse module to improve prediction accuracy. The discourse feature extraction relies on the same automatic speech recognition module as described in the lexical features (Lexical features).

\section{Classifiers}

The classifiers form the core component of the framework, which is used to provide QoE prediction based on an input set of features extracted from previous feature extraction modules (shown in Figure 2). The original data set contains samples tagged with user quality feedback, which is divided into training set and testing set. For the initial training phase, samples of the training set are utilized to build a classifier. During the testing phase, samples of the testing set are fed into the classifier (with user feedback removed) which will output QoE predictions. Then, the output is used to compare with the user feedback. Overall, $75 \%$ of the data samples are used for training and the rest $25 \%$ for testing.

Two basic types of classifiers are used here, namely Support Vector Machines (SVM) and $k$-Nearest Neighbors (kNN). SVM involves the construction of a set of hyperplanes by maximizing the separating distance between the nearest training data points among all classes. In contrast, $k \mathrm{NN}$ works by computing the $k$ nearest neighbors to the input sample based on a distance metric (by default, Euclidean) and using a majority vote among the neighbors to determine the class label of the sample. Our rationale for choosing SVM and $k \mathrm{NN}$ classifiers are mainly due to their effectiveness and performance benefit. Though we test various classification methods, our framework does not depend on any one particular technique.

We make no assumption on the dependency between these features and the user experience of quality. Instead, we take an unequivocal classification approach, using the ground truth from the training data. For SVM classifier, we trained our models 
with the radial basis function (RBF) kernel. To identify the optimal values of $\mathrm{C}$ (cost) and $\Upsilon$ (kernel parameter), we apply cross-validation with iteratively refined grid-search method [30]. The notations of the four different SVM derivatives used in our study are given as follows.

- $S V M$ : uses fixed training and testing sets.

- $S V M-5 C V$ : divides the data into five randomly chosen segments of equal size and run five times with each run comprising of four segments for training and one segment for testing (5-fold cross-validation).

- SVM-5WC and SVM-10WC: employs 2-layer hierarchical SVM model that trains 5 and 10 bottom-layer weak classifiers ( $5 \mathrm{WC}$ and $10 \mathrm{WC}$ respectively) on different subset of the training set and the output of each is used to train a top-layer meta-classifier.

For $k N N$ classifier, we trained two derivatives. Their notations are given as follows.

- $k N N$ : similar to that of $S V M$ with $k=10$.

- $k N N-5 C V$ : similar to that of $S V M-5 C V$ with an iterative number of nearest neighbors from $k=1$ to 15 .

Since we incorporate multiple affective sources (i.e., acoustic, lexical, discourse) in our framework, we need aggregation scheme to produce a single output. One simple method is to employ feature-level fusion which merges all features from different sources into a large feature vector as the input to a single classifier. In contrast, decision-level fusion takes results from multiple classifiers to compute a single value. We take the second scheme and calculate the average of results from each classifier as the final output. As simple as it is, such method achieves pretty good performance $[54,55]$. For software, we use the tools of libSVM [56] for SVM classifiers and Weka eweka for $k \mathrm{NN}$ classifiers.

\section{Experimental design}

We designed the user study experiment for the examination of the following research hypothesis:

$\mathrm{H}$ : The user perception of voice communication quality is correlated to his/her affective response, which will vary across networking conditions.

The main purpose of the experiment was to collect two types of data: audio signal of user conversation and the user quality feedback. For that purpose, we engaged two participants in natural conversation through a VoIP connection. During thetest, we tuned the QoS parameters of delay, loss rate and bandwidth to simulate various networking conditions. We expect that the affect state of user as reflected from his/her voice will change accordingly, as well as his/her subjective perception of the communication quality. Thus, we recorded the voice conversation and asked the user to rate the quality. The collected data were used for training and testing by the affect analysis framework in the next stage (Related work).

\section{Networking}

We installed two desktop computers with Intel i686 Core 2 Quad CPU 32-bit (2.39 GHz) and 2 GB RAM running Linux kernel (Ver. 2.6.31.5) as each end of a VoIP connection. The two computers were placed in two separate and quiet rooms of our department building (one in the second floor and the other in the third floor). During 
the experiment, no other person was allowed inside the room to avoid any psychological influence on the participants.

We configured a third computer (with similar hardware settings and the same operating system) to be used as a layer-2 bridge. The bridge computer had two network interface cards to connect with the two VoIP end computers. We installed the Linux brctl utility on the bridge computer to form a LAN: (VoIP end computer 1) $\leftrightarrow$ (bridge computer $) \leftrightarrow$ (VoIP end computer 2). All physical connections were based on dedicated wired lines with no interference from external network traffic. To simulate various networking conditions, we instrumented the traffic flow between the two VoIP end computers by applying dummynet [57] on the bridge computer. The dummynet software allows us to tune the network condition with different delay, loss rate and bandwidth settings.

\section{Voice channel}

Each VoIP end computer was equipped with a Logitech headset with speaker/microphone for voice capturing, playback, and recording. We used the library of PortAudio [58] to record audio signals from the microphone into wav files that can be further processed by the feature extraction modules. For simplicity, each end only records its local signal for QoE evaluation. The interplay between local and remote signals would be an interesting topic for future research. We deployed PJSIP [59] as the VoIP software with G.711 codec. It is an open-source, comprehensive, and highly portable system with a small memory footprint. Although PJSIP contains lots of features, it is console-based and the basic command set is very simple. After a few minutes' instruction, all participants were capable of operating it. To facilitate the experiment, we automated the communication and session management process for call initiation, connection, and recording, so that two end users only need to press a few keys to launch a test session.

\section{Sample collection}

We organized each test session based on a maximum 15-min run. The limit here was chosen basically due to our observation that it was not natural to engage two strangers in a phone conversation for over-stretched duration. Thus, to improve the efficiency of every test we divided a run into multiple 20 -s intervals. Timescale of each interval is an important issue for QoE evaluation which is justified in Timescale. Each interval corresponded to a fixed setting of QoS parameters. We adopted the approach of OneClick [15] in the sense that at the end of each interval a console prompt was shown to ask for user input of quality. Different from OneClick, we employed a trichotomous or 3-point scale decision ofquality levels: "Good", "Average", and "Bad". Although this experimental methodology still bears some intrusiveness, it is unavoidable in the initial study since we need user feedback for training and testing the affect analysis framework. The complete establishment of the framework will eventually eliminate the need of user feedback.

The interval was saved as one sample after it was tagged with QoS parameters and user feedback. Then, a different set of QoS parameters was configured into the network and the next interval was started (shown in Figure 3). All the stepswere automatically synchronized. Besides pressing a key every $20 \mathrm{~s}$, there was no extra distraction 


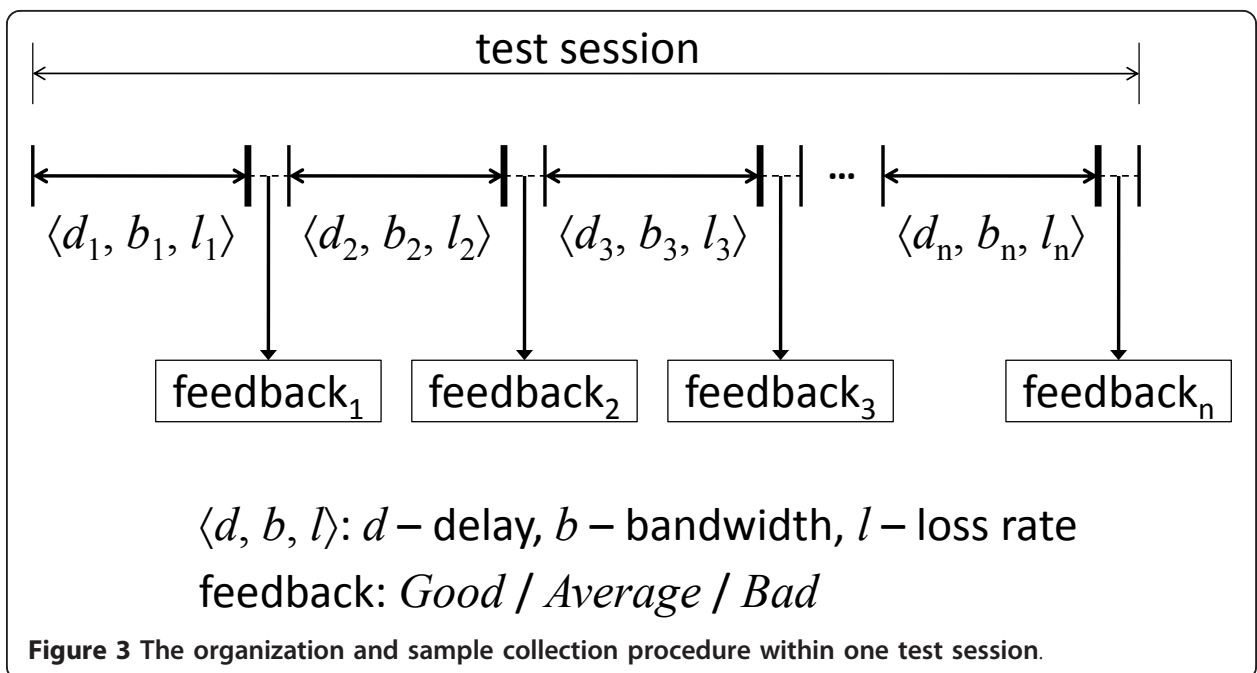

to the end user. Overall, one test run provided 90 samples (i.e., 45 samples from each end).

\section{Timescale}

It has been recognized that user perception of speech quality varies under different temporal scale [46]. For example, in short-term test $(\approx 30$ seconds) the beginning portion (at least $>8$ seconds) of the speech sample carries the greatest weight in the overall rating of MOS (primary effect) [60], while in long-term test ( $\geq 60$ seconds) the last portion carries the greatest weight (recency effect) [61]. Regarding its psychological merit of human memory mechanism, these findings give us the guideline for deciding the timescale of test interval. Because user feedback is requested at the end of each interval, we should choose short duration for each interval to leverage on primary effect (but not too short). On the other hand, since the QoS parameters are fixed in one interval, there is no need to apply long duration due to the recency effect. Considering previous research results and the possible interference to the user, we chose $20 \mathrm{~s}$ as the fixed timescale for each test interval. ${ }^{\mathrm{a}}$

\section{QoS classes}

We employed delay $(d)$, bandwidth $(b)$ and loss rate $(l)$ as the basic QoS parameters. For notation, each networking configuration is denoted as a tuple of $\langle d, b, l\rangle$. Because the space of possible configurations isquite large, we applied a selection procedure to pick the most "distinctive"value set. In the pilot experiment, we performed the empirical study to pick up a meaningful range for each parameter. First, we set the best configuration as gle $50 \mathrm{~ms}, 100 \mathrm{Kbps}, 0.06>$. Then the worst configuration of each parameter was determined incrementally while keeping the other two as the best until the quality became intolerable. In such a way, the worst delay was set as $1200 \mathrm{ms,}$ worst bandwidth $52 \mathrm{Kbps}$, and worst loss rate 0.3. Next, we picked 5 values from each parameter range evenly and aligned them together from best to worst and generated 5 $\mathrm{Q}$ o $\mathrm{S}$ classes.

- $C_{1}:<d=50 \mathrm{~ms}, b=100 \mathrm{Kbps}, l=0.06>$

- $C_{2}:<d=300 \mathrm{~ms}, b=88 \mathrm{kbps}, l=0.12>$ 
- $C_{3}:<d=600 \mathrm{~ms}, b=76 \mathrm{kbps}, l=0.18>$

- $C_{4}:<d=900 \mathrm{~ms}, b=64 \mathrm{kbps}, l=0.24>$

- $C_{5}:<d=1200 \mathrm{~ms}, b=52 \mathrm{kbps}, l=0.3>$

For further validation, we asked a few graduate students to run MOS-based test to rate the quality for each configuration. The test was done in a random and blind way (i.e., the students were not aware of the configuration). The average rating resultsconfirmed clearly the quality difference between each configuration.

\section{Participants}

We recruited 15 participants from two mid-level undergraduate classes, namely, Java Programming and Computer Networks [1]. We found that selecting students from same classes provided better conversation control, for example, the familiarity and the common interest (details in Conversation). All students were proficient with English language (6 native, 7 advanced and 2 intermediate speakers). Among them, 11 were male and 4 were female, between $18-38$ years of age $(M=25.54, S D=3.38)$. They had a mean of 3.67 years of using VoIP service and all claimed to have been using at least one VoIP service in the past (with the most popular being Skype followed by GTalk).

For better control of the test (more in Conversation) and regarding the difficulty of accommodating each participant's schedule, in each test session a graduate student was assigned to play one VoIP end with another undergraduate student at the other end (i.e., the real participant). So there were totally 15 test sessions. For statistic validity, the samples from the graduate student were discarded. Participation in the experimental study was considered as a benefit by the students and no monetary compensation was involved, since the conversation topics were chosen from course materials as discussed later.

\section{Questionnaire}

Before the experiment, each participant was introduced with the experimental details following IRB required procedures. Then he/she was asked to complete an Entry Questionnaire at the beginning of the study for collecting background/demographic information, and their previous experience with VoIP if any. Exit Questionnaires were also provided at the end of each session to elicit subjective experience of the user for the entire audio conversation. The questionnaire gathered information on the subjective quality perception and the evaluation criterion, as well as the participant's view of the importance of affective feedback regarding the usability and ethical outcome (for IRB purposes). Some of the questions are listed below:

Q: What is your overall perception of the system?

Q: Did the system perform as per your expectation?

Q: Were you able to interact with the system without any problems?

Q: Did you feel any external or environmental influence during the experiments?

Q: Did you expect any more help from the system?

\section{Conversation}

Since conversational materials are highly correlated to the quality metrics [62], a careful design of dialog exchange is important for the experiment. We wanted to invoke natural conversation from the participants. Initially, we thought about selecting a few 
topics. However, the cultural and emotional influence of the topic on each user would be hard to predict and may interfere with his/her experience. The ITU recommendation also suggests that the content should be "neither so interesting nor so disagreeable or boring" [63]. To make the communication more controllable, we decided to give quiz-like conversation formed using course-related materials with low-level difficulty. The graduate student (actually the TA of the class) presented some questions and discussed with the student. This kind of conversation can be easily engaged and the topic is neutral. Moreover, to avoid over-burdening the participants, we embedded some brief comments on general topics or short riddles from time to time to provide cognitive relief.

It is observed that in some cases the conversation itself might have invoked emotional responses that are not related to communication quality perception. In the experiment, we attempted to reduce this kind of effect by preferring neutral conversational content as described. However, in the real scenario, people do engage with heated conversational dialog among themselves. In our preliminary experiment, we tried to filter out "emotional noise" from the perspective of conversational content by considering lexical and discourse features such as salient words and repetitions. Note that, such drawback is inherent in any type of subjective assessment including MOS. This challenging issue deserves further study which is the focus of our future work.

\section{Results}

In this section we present the experimental results of our study based on test sessions collected from 15 subjects. One finding of interest is the performance of QoE prediction where we compared the output from the affect-based framework with the user feedback (both on a 3-point scale of quality levels). We also highlight other results and implications regarding the interaction between QoS setting, user feedback and quality prediction.

\section{Performance of estimation}

We conducted a comprehensive study of estimation accuracy along three dimensions as introduced in Affect analysis framework: (1) different classifiers of SVM and $k \mathrm{NN}$, (2) different combinations of affective sources (acoustic: A, lexical: $\mathrm{L}$ and discouse: D), and (3) different feature selection schemes (Base, f10, f15, and PCA), which are shown in Figure 4. The overall findings are summarized as follows: $(a)$ combining other affective sources with acoustic features consistently improves the performance where the best results appear from the aggregation of all sources $(A+L+D),(b)$ considering single source, QoE prediction based on acoustic source is more accurate than the other two whereas the performance of lexical source is slightly better than that of discourse source, and $(c)$ the performance impact of different feature selection schemes is less noticeable in $k \mathrm{NN}$ classifiers than SVM classifiers.

Table 1 shows the cross-view of the results based on all affective sources $(\mathrm{A}+\mathrm{L}+$ D) with different feature selection schemes and classifiers. In all cases, the PCA feature selection scheme provides the highest performance. The $f 10$ and $f 15$ feature selection schemes provide comparable accuracy which is consistently higher than the base feature set. Comparing different classifiers, it is observed that SVM with 5-fold cross-validation ( $S V M-5 C V)$ gives the highest accuracy. As a short summary, the best 


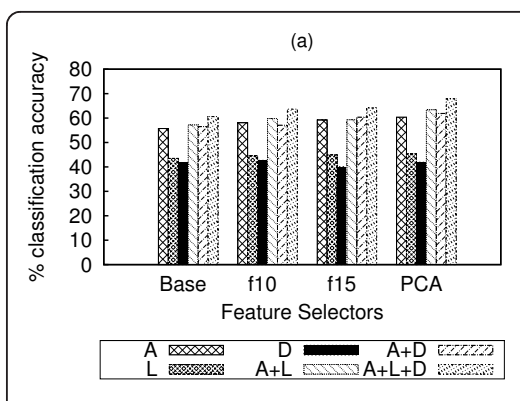

(c)
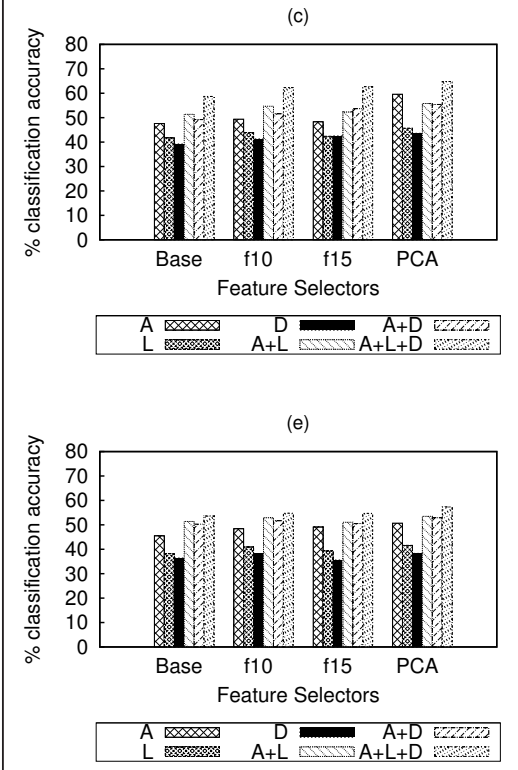
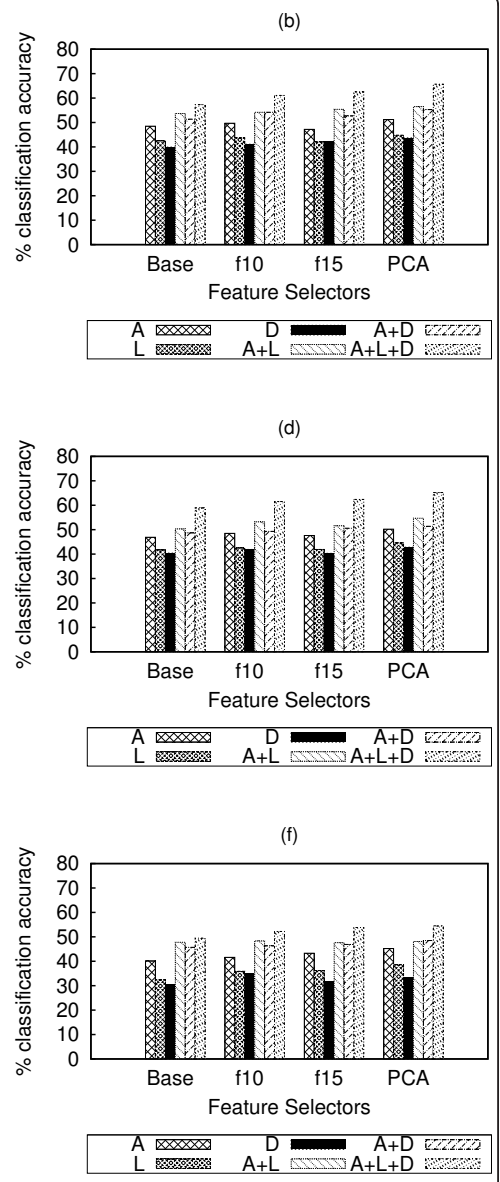

Figure 4 Classification accuracy for different combinations of affective sources $(A=A c o u s t i c, L=$ Lexical, $D=$ Discourse), feature selection schemes (Base, f10,f15, PCA) and different classification techniques of (a) xtitSVM, (b) SVM-5WC, (c) SVM-10WC, (d) SVM- 5CV, (e) kNN, and (f) kNN-5CV.

performance is achieved with SVM 5-fold cross-validation and PCA-based feature selection, which gives an accuracy of $67.9 \%$.

\section{OoS distribution}

We next examine the distribution of QoS classes among various user quality ratings. Recall that, each conversation interval is initialized by applying a particular QoS class (i.e., $\mathrm{C}_{1}, \mathrm{C}_{2}, \mathrm{C}_{5}$, QoS Classes) to set the networking condition and a quality rating is recorded from the user feedback at the end of theinterval. Figure 5 shows the

Table 1 Classification accuracy with different feature sets versus varying classifiers from the combination of all affective sources $(A+L+D)$

\begin{tabular}{lllll}
\hline Classifier Model & Base & $\mathbf{f 1 0}$ & $\mathbf{f 1 5}$ & PCA \\
\hline SVM & 57.3 & 61.1 & 62.5 & 65.7 \\
\hline SVM-5WC & 58.7 & 62.3 & 62.7 & 64.8 \\
\hline SVM-10WC & 58.9 & 61.5 & 62.4 & 65.2 \\
\hline SVM-5CV & 60.6 & 63.7 & 64.1 & 67.9 \\
\hline kNN & 49.7 & 52.8 & 53.1 & 54.3 \\
\hline kNN-5CV & 53.5 & 54.2 & 54.9 & 57.4
\end{tabular}




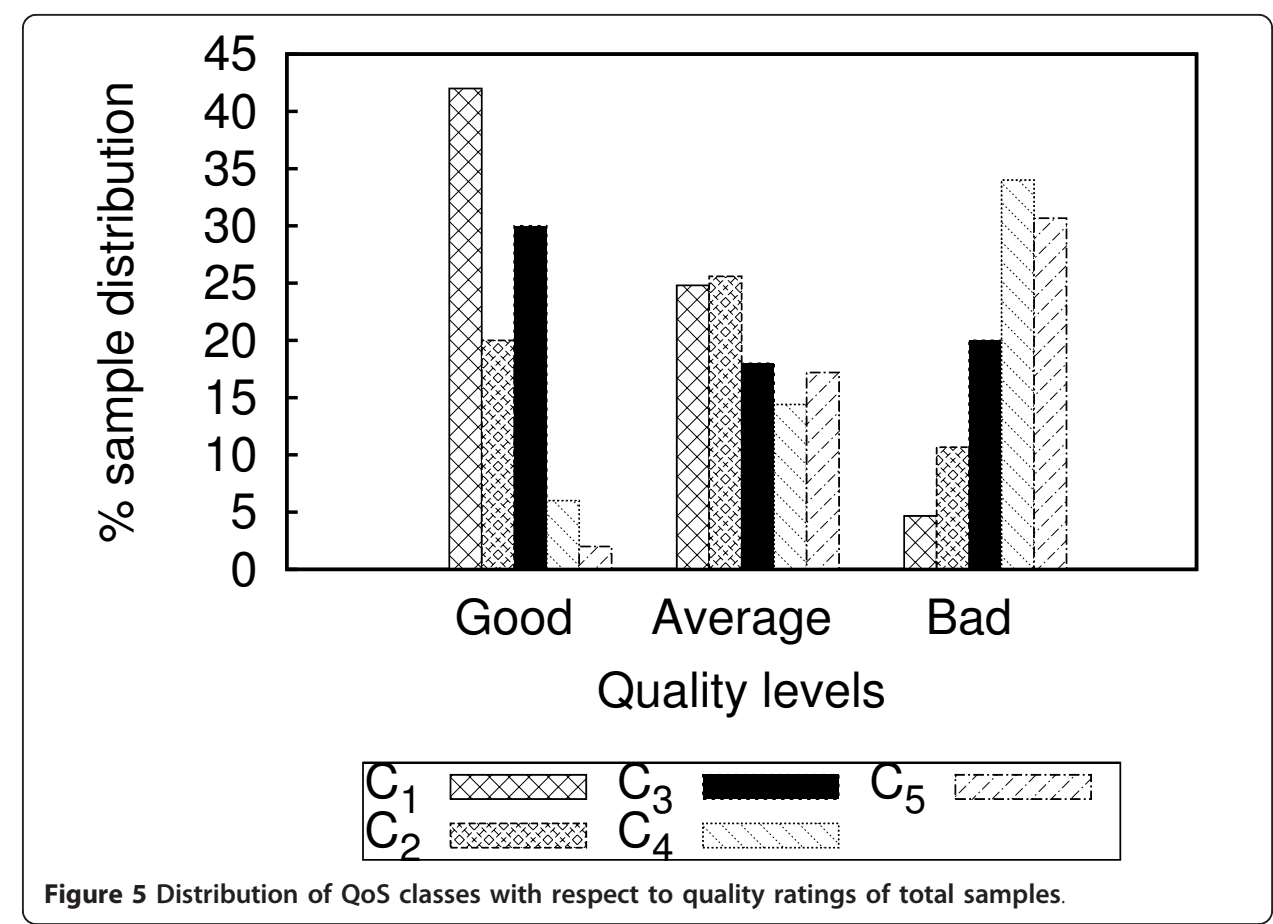

distribution of the five different QoS classes with respect to the user feedback for "Good", "Average", and "Bad" quality ratings. As seen, the highest portion of "Good" quality rating is associated with QoS class $C_{1}(42.0 \%)$, and the overall trend is decreasing from $C_{1}$ to $C_{5}$. However, quite interestingly the results also show that $C_{3}$ contributes morefor "Good" quality rating (30.0\%) in comparison with $C_{2}(20.0 \%)$. In a similar way, $C_{4}$ takes the highest distribution for " $B a d^{\text {" }}$ quality rating (34.0\%) which is more than $C_{5}$ (30.67\%). For the "Average" case, the quality rating is more evenly distributed across all QoS classes. Although more rigorous study is still needed, the finding of our preliminary study does imply that the correspondence between different quality ratings and networkQoS classes does not always bear a close coherent relationship. This seems to validate our previous observation that solely QoS-based estimation is not sufficient for QoE prediction as discussed in related work.

\section{Correlation in QoS classes}

From another angle, the results of correlation between the user quality feedback and the quality prediction are given for each QoS class in Table 2 using different calculation methods (Pearson, Kendall and Spearman). It is observed that different network QoS classes have varied correlation impact: QoS classes of $C_{1}$ and $C_{5}$ demonstrate the highest correlation, $C_{2}$ and $C_{4}$ stay as the intermediate cases, while $C_{3}$ has the lowest correlation. The implication wecan draw here is that QoE prediction tends to be more accurate if the underlying networking condition is either very good or very bad, and becomes less accurate for mid-range networking conditions. The finding also suggests that we should be more careful to apply QoS-based estimation. The usage of such methods need to take into account of different QoS contexts (e.g., extreme vs. average conditions). 
Table 2 Correlation tests of the quality prediction from the testing samples

\begin{tabular}{llll}
\hline Selection & Pearson & Spearman & Kendall \\
\hline$C_{1}$ & 0.8068 & 0.7856 & 0.784 \\
\hline$C_{2}$ & 0.5424 & 0.5338 & 0.507 \\
\hline$C_{3}$ & 0.1026 & 0.0871 & 0.0752 \\
\hline$C_{4}$ & 0.5572 & 0.4766 & 0.4743 \\
\hline$C_{5}$ & 0.7321 & 0.7319 & 0.7325 \\
\hline Overall & 0.5697 & 0.5351 & 0.5608 \\
\hline
\end{tabular}

Implication of quality ratings

Finally, we examine the interaction between user quality ratings and the predictions made by the affect analysis framework. We refer to the results of $S V M-5 C V$ only since it is the classifier of the best performance as observed in Section fsubsec:accuracy. The testing samples are plotted in Figure 6 which shows the values of user quality rating as well as the prediction. For better visualization, the testing samples are ordered with respect to the user quality ratings from "Good", "Average", to "Bad."

Table 3 summarizes the number of user ratings in each quality level and the corresponding prediction accuracy. Some of the observations from the table are as follows: (a) though the number of "Good" quality ratings are low but it still has a high degree

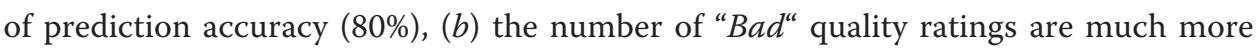
in number with a comparable degree of prediction accuracy $(77.78 \%)$, and $(c)$ the "titAverage" quality ratings have a relatively low degree of accuracy (58.67\%) compared to the other two but it has the most number of cases. The study shows that quality rating of "Average" seems to be more ambiguous than the ratings of"Good" and "Bad." The reason may be that people tend to rate "Average" more frequently across different network conditions (also shown in other studies as well).

\section{Conclusions}

We have presented a user study experiment to evaluate an affect-based approach of QoE evaluation in voice communication, which represents a new and unexplored area. The purpose of the study is to examine how user affective behavior changes with the communication quality as mediated through different network QoS conditions, and how such changes can be detected and used to predict QoE from the user perspective. We evaluated the effectiveness of this approach by using classification techniques based on SVM and kNN to discriminate different quality perceptions and compare with user feedback. The accumulated evidence supports our initial hypothesis of

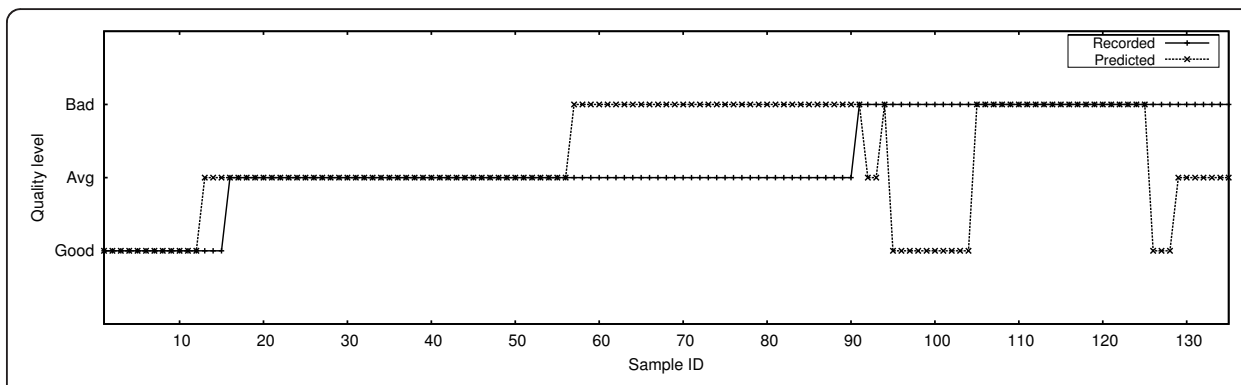

Figure 6 Comparison of the distribution of different quality levels for the testing samples with respect to the user feedback and predicted values for SVM-5CV. 
Table 3 Correlation tests of the quality prediction from the testing samples

\begin{tabular}{lll}
\hline User Quality Rating & Number of cases & Accuracy (\%) \\
\hline "Good" & 15 & 80.0 \\
\hline "Average" & 75 & 58.7 \\
\hline "Bad" & 45 & 77.8 \\
\hline
\end{tabular}

exploiting affective responses as the predictor of subjective experience of quality due to its correlation with human cognitive perception. Our best performance achieves a prediction accuracy of $67.9 \%$. Although we refrain from claiming that our methodology can cover the entire spectrum of QoE evaluation factors, our study provided contributory illustrations for affective information to be considered as a relevant indicator. Since our work represents the first attempt in this area, a cross comparison with related existing approaches will be difficult. For example, our system is not directly comparable to QoS-based estimation methods because the latter provides objective measures. The QoE estimation will be the same for one single QoS setting which ignores the variation of the user group. We are aware that our present study still has certain limitations such as the potential emotional influence. It seems likely that in some cases the conversation itself might have invoked emotional responses that are not related to communication quality perception. We are presently working on this aspect to filter out the "emotional noise."

We plan to continue our current research along the following possible directions in the future: (a) to study other affective cues (e.g., laughter and sigh) on the subjective quality of experience, $(b)$ to integrate other discourse related attributes (e.g., rephrase, reject and ask over) in the present framework for further evaluation, $(c)$ to improve the implementation for real-time processing, and $(d)$ to investigate an integration of both subjective and objective QoE evaluation methods. Finally, our testing method applies QoS settings to the communication channel in a random fashion, which could be improved by following real Internet traces to simulate a more realistic testing environment $[64,65]$.

\section{Endnote}

${ }^{a}$ The ITU-T recommendation for the subjective assessment of sound quality also suggests the clip duration from 15 to $20 \mathrm{~s}$, and the minimum number of participants in an experiment to be between 10 and 20 [63].

\section{Acknowledgements}

This study is supported by the National Science Foundation (NSF) under CSR-1116023. The presented views are those of authors and do not represent the position of NSF.

\footnotetext{
Author details

${ }^{1}$ School of Computing and Information Sciences, Florida International University, Miami, FL 33199, USA ${ }^{2}$ Department of Computer Science, University of Illinois at Urbana-Champaign, Urbana, IL 61801, USA
}

Authors' contributions

$A B$ carried out the framework-experiment design, implementation, experimental data collection and analysis, and manuscript drafting phases of the above work. WW participated in the high-level design, manuscript drafting, and the analysis phase of the work. ZY was involved with the design, experimental analysis, and manuscript editing phase of the work. All authors read and approved the final manuscript.

Competing interests

The authors declare that they have no competing interests. 


\section{References}

1. Jaimes A, Sebe N, Gatica-Perez D (2006) Human-centered computing: a multimedia perspective. ACM international conference on Multimedia. MULTIMEDIA '06, New York http://doi.acm.org/10.1145/1180639.1180829

2. Jain R (2004) Quality of experience. Multimedia IEEE 11

3. Tasaka S, Yoshimi H, Hirashima A, Toshiro N (2008) The effectiveness of a QoE-based video output scheme for audiovideo ip transmission. ACM international conference on Multimedia. MM '08, New York http://doi.acm.org/10.1145/ 1459359.1459395

4. Ebrahimi T (2009) Quality of multimedia experience: past, present and future. ACM international conference on Multimedia. MM '09, New York http://doi.acm.org/10.1145/1631272.1631275

5. Subjective audiovisual assessment methods for multimedia experience. Tech. rep., ITU-T Rec (1998) 911

6. Beauregard R, Corriveau P (2007) User experience quality: a conceptual framework for goal setting and measurement. International conference on Digital human modeling. ICDHM'07, Springer-Verlag, Berlin http://portal.acm.org/citation. $\mathrm{cfm}$ ?id=1784074.1784114

7. Wu W, Arefin A, Rivas R, Nahrstedt K, Sheppard R, Yang Z (2009) Quality of experience in distributed interactive multimedia environments: toward a theoretical framework. ACM international conference on Multimedia. MM '09, New York http://doi.acm.org/10.1145/1631272.1631338

8. Forlizzi J, Battarbee K (2004) Understanding experience in interactive systems. Conference on Designing interactive systems: processes, practices, methods, and techniques. DIS '04, New York http://doi.acm.org/10.1145/1013115.1013152

9. Csikszentmihalyi M (1990) Flow: The psychology of Optimal Experience. Harper and Row

10. Varela F, Thompson E, Rosch E (1992) The Embodied Mind: Cognitive Science and Human Experience. The MIT Press

11. Hobbs D, Stuart B (1982) Sociology and the human experience. Wiley

12. Abowd G, Mynatt E, Rodden T (2002) The human experience [of ubiquitous computing]. Pervasive Computing, IEEE

13. Davis FD, Bagozzi RP, Warshaw PR (1989) User acceptance of computer technology: a comparison of two theoretical models. Manag Sci 35(8)http://www.jstor.org/stable/2632151

14. Venkatesh V, Morris MG, Davis GB, Davis FD (2003) User acceptance of information technology: toward a unified view. MIS Quarterly 27(3)http://www.jstor.org/stable/30036540

15. Chen KT, Tu CC, Xiao WC (2009) OneClick: a framework for measuring network quality of experience. INFOCOM 2009 IEEE

16. ITU (1996) ITU-T Recommendation P.800:Methods for subjective determination of transmission quality. Geneva

17. Chen KT, Huang CY, Huang P, Lei CL (2006) Quantifying Skype user satisfaction. Proceedings of the 2006 conference on Applications, technologies, architectures, and protocols for computer communications. SIGCOMM '06, New York

18. Sat B, Wah BW (2007) Playout scheduling and loss-concealments in voip for optimizing conversational voice communication quality. ACM International conference on Multimedia. MULTIMEDIA '07, New York http://doi.acm.org/ $10.1145 / 1291233.1291260$

19. ITU (2001) Perceptual evaluation of speech quality (PESQ): An objective method for end-to-end speech quality assessment of narrow-band telephone networks and speech codes. Geneva

20. ITU (2005) ITU-T Recommendation G.107. The E-model, a computational model for use in transmission planning. Geneva

21. Nahl D (2004) Measuring the affective information environment of web searchers. American society for information science and technology $41 \mathrm{http}: / / d x . d o i . o r g / 10.1002 /$ meet.1450410122

22. Nahl D (1998) Learning the Internet and the structure of information behavior. J Am Soc Inf Sci 49http://portal.acm.org/ citation.cfm?id=288129.288148

23. Tenopir C, Wang P, Zhang Y, Simmons B, Pollard R (2008) Academic users' interactions with sciencedirect in search tasks: affective and cognitive behaviors. Inf Process Manage 44http://portal.acm.org/citation.cfm?id=1314720.1315078

24. Kracker J (2002) Research anxiety and students' perceptions of research: an experiment. Part I: Effect of teaching Kuhlthau's ISP model. J Am Soc Inf Sci Technol 53http://portal.acm.org/citation.cfm?id=507286.507289

25. Zeng Z, Pantic M, Roisman G, Huang T (2009) A survey of affect recognition methods: audio, visual, and spontaneous expressions. Pattern Analysis and Machine Intelligence, IEEE Transactions on 31

26. Kapoor A, Picard RW (2005) Multimodal affect recognition in learning environments. ACM international conference on Multimedia. MULTIMEDIA '05, New York http://doi.acm.org/10.1145/1101149.1101300.

27. Pelachaud C (2005) Multimodal expressive embodied conversational agents. ACM international conference on Multimedia. MULTIMEDIA '05, New York http://doi.acm.org/10.1145/1101149.1101301.

28. Calvo R, D'Mello S (2010) Affect detection: an interdisciplinary review of models, methods, and their applications. IEEE Trans Affect Comput 1

29. Arapakis I, Jose JM, Gray PD (2008) Affective feedback: an investigation into the role of emotions in the information seeking process. ACM SIGIR conference on Research and development in information retrieval. SIGIR '08, New York

30. Arapakis I, Konstas I, Jose JM (2009) Using facial expressions and peripheral physiological signals as implicit indicators of topical relevance. ACM international conference on Multimedia. MM '09, New York

31. Vinciarelli A, Pantic M, Bourlard $H$, Pentland A (2008) Social signal processing: state-of-the-art and future perspectives of an emerging domain. ACM international conference on Multimedia > MM '08, New York http://doi.acm.org/10.1145/ 1459359.1459573

32. Vinciarelli A, Pantic M, Bourlard H (2009) Social signal processing: Survey of an emerging domain. Image Vision Comput 27:1743-1759http://dl.acm.org/citation.cfm?id=1621144.1621310. doi:10.1016/j.imavis.2008.11.007.

33. Curhan JR, Pentland A (2007) Thin slices of negotiation: predicting outcomes from conversational dynamics within the first 5 minutes. J Appl Psychol 92(3):802-811http://www.sciencedirect.com/science/article/pii/S0021901007670058

34. Cowie R, Douglas-Cowie E, Tsapatsoulis N, Votsis G, Kollias S, Fellenz W, Taylor J (2001) Emotion recognition in humancomputer interaction. Signal Processing Magazine, IEEE 18

35. Batliner A, Fischer K, Huber R, Spilker J, Nöth E (2003) How to find trouble in communication. Speech Commun 40http://portal.acm.org/citation.cfm?id=772592.772600 
36. Lee CM, Narayanan S (2005) Toward detecting emotions in spoken dialogs. IEEE Transactions on Speech and Audio Processing 13(2)

37. Knoche H, Meer HGD, Kirsh D (1999) Utility curves: mean opinion scores considered biased. International conference on Quality of Service. IWQOS'99

38. Watson A, Sasse MA (1998) Measuring perceived quality of speech and video in multimedia conferencing applications. ACM international conference on Multimedia. MULTIMEDIA '98, New York http://doi.acm.org/10.1145/290747.290755

39. Markopoulou A, Tobagi F, Karam M (2002) Assessment of VolP quality over Internet backbones. INFOCOM 2002. Twenty-First Annual Joint Conference of the IEEE Computer and Communications Societies. Proceedings. IEEE 1

40. Wu CC, Chen KT, Huang CY, Lei CL (2009) An empirical evaluation of VolP playout buffer dimensioning in Skype, Google talk, and MSN Messenger. International workshop on Network and operating systems support for digital audio and video. NOSSDAV '09, New York

41. Graubner M, Mogre PS, Steinmetz R, Lorenzen T (2010) A new QoE model and evaluation method for broadcast audio contribution over IP. Internatio nal workshop on Network and operating systems support for digital audio and video. NOSSDAV '10, New York http://doi.acm.org/10.1145/1806565.1806581

42. Chen KT, Wu CC, Chang YC, Lei CL (2009) A crowdsourceable QoE evaluation framework for multimedia content. ACM international conference on Multimedia. MM '09, New York http://doi.acm.org/10.1145/1631272.1631339

43. Baset SA, Schulzrinne HG (2006) An analysis of the skype peer-to-peer internet telephony protocol. IEEE International Conference on, Computer Communications

44. Bonfiglio D, Mellia M, Meo M, Rossi D, Tofanelli P (2007) Revealing skype traffic: when randomness plays with you. Proceedings of the 2007 conference on Applications, technologies, architectures, and protocols for computer communications. SIGCOMM '07, New York http://doi.acm.org/10.1145/1282380.1282386

45. Falk T, Chan WY (2006) Single-Ended speech quality measurement using machine learning methods. Audio, Speech, and Language Processing, IEEE Transactions on 14(6)

46. Rix A, Beerends J, Kim DS, Kroon P, Ghitza O (2006) Objective assessment of speech and audio quality - technology and applications. IEEE Trans Audio Speech Language Process 14(6)

47. Eyben F, Wöllmer M, Schuller B (2010) OpenSMILE: the munich versatile and fast open-source audio feature extractor. ACM international conference on Multimedia. MM '10, New York http://doi.acm.org/10.1145/1873951.1874246

48. Gorin A, Levinson S, Miller L, Gertner A, Ljolje A, Goldman E (1990) On adaptive acquisition of language. Acoustics, Speech, and Signal Processing, 1990. ICASSP-90., 1990 International Conference on 1

49. Woodland P, Odell J, Valtchev V, Young S (1994) Large vocabulary continuous speech recognition using HTK. Acoustics, Speech, and Signal Processing, 1994. ICASSP-94., 1994 IEEE International Conference on, Volume ii. II/125 -II/128 2

50. Vertanen K Baseline wsj acoustic models for htk and sphinx: training recipes and recognition experiments.

51. English gigaword linguistic data consortium, Philadelphia.http://www.ldc.upenn.edu/Catalog/CatalogEntry.jsp? catalogld =LDC2003T05

52. Ang J, Dhillon R, Krupski A, Shriberg E, Stolcke A (2002) Prosody-based automatic detection of annoyance and frustration in human-computer dialog. ICSLP

53. Interactions ICM, Arunachalam S, Gould D, Andersen E, Byrd D, Narayanan S (2001) Politeness and frustration language. Eurospeech

54. Kittler J, Hatef M, Duin R, Matas J (1998) On Combining Classifiers. IEEE Transactions on Pattern Analysis and Machine Intelligence 20(3)

55. Tax DMJ, van Breukelen M, Duin RPW, Kittler J (2000) Combining multiple classifiers by averaging or by multiplying? Pattern Recognit 33(9)http://www.sciencedirect.com/science/article/B6V14-40961WK-7/2/ 9624dda26ddc086458febdde9f819862

56. Chang CC, Lin CJ LIBSVM: a library for support vector machines.http://www.csie.ntu.edu.tw/cjlin/libsvm

57. Rizzo L (1997) Dummynet: a simple approach to the evaluation of network protocols. SIGCOMM Comput Commun Rev 27http://doi.acm.org/10.1145/251007.251012

58. PortAudio: Portable Cross-Platform Audio I/O.http://www.portaudio.com

59. PJSIP: Open source SIP stack and media stack for presence, instant messaging, and multimedia communication.http:// www.pjsip.org

60. Gray P, Massara R, Hollier M (1997) An experimental investigation of the accumulation of perceived error in timevarying speech distortions. Audio Engineering Society Convention 103http://www.aes.org/e-lib/browse.cfm?elib=7191

61. Rosenbluth JH (1998) Testing the quality of connections having time varing impairments. ITU-T del cont COM12-D64

62. Woodruff A, Aoki PM (2004) Conversation analysis and the user experience. ACM SIGCHI workshop on exploring experience methods across disciplines

63. ITU (2003) ITU-T Rec. BS.1284-1: General methods for the subjective assessment of sound quality. Geneva

64. CMU pronounciation dictionary.http://www.speech.cs.cmu.edu/cgi-bin/cmudict

65. Hall M, Frank E, Holmes G, Pfahringer B, Reutemann P, Witten IH (2009) The WEKA data mining software: an update. SIGKDD Explor Newsl 11http://doi.acm.org/10.1145/1656274.1656278

doi:10.1186/2192-1962-2-7

Cite this article as: Bhattacharya et al:: Quality of experience evaluation of voice communication: an affect-based

approach. Human-centric Computing and Information Sciences 2012 2:7. 